Themenheft Nr. 46: Eltern - Pädagog*innen - Medienkompetenzen.

Eltern und Pädagog*innen zwischen Aneignung und Vermittlung von Medienkompetenz. Herausgegeben von Thorsten Naab, Alexandra Langmeyer, Ruth Wendt und Jessica Kühn

\title{
Wie verändert sich Medienerziehung im familienbiografischen Verlauf?
}

\section{Eine quantitative längsschnittliche Analyse zur Stabilität elterlicher Medienerziehung aus Sicht von Eltern und Heranwachsenden}

\author{
Katrin Potzel ${ }^{1}$ (D) \\ ${ }^{1}$ Friedrich-Alexander-Universität Erlangen-Nürnberg
}

\begin{abstract}
Zusammenfassung
Eine längerfristige Betrachtung elterlicher Medienerziehung findet noch kaum Beachtung. Ziel des vorliegenden Beitrags ist es Parental Mediation Strategien (aktive, restriktive und technische Mediation, ebenso wie Monitoring) empirisch über einen längeren Zeitraum zu untersuchen. Ein multiperspektivischer Ansatz ermöglicht einen Vergleich zwischen Eltern- und Jugendlichenperspektive. Eine besondere Rolle spielt zudem die Frage, wie konsequent sich Eltern in diesen Erziehungsstrategien verhalten und ob ebenfalls zeitliche Veränderungen beobachtbar sind. Die quantitative Panelstudie «Verläufe exzessiver Internetnutzung in Familien (VEIF)» liefert über vier Erhebungswellen im Abstand von etwa einem Jahr Daten aus Eltern- und Jugendlichensicht. So kann durch die Auswertung ein multiperspektivischer und längsschnittlicher Einblick gewonnen werden. Die Heranwachsenden waren zu Beginn zwischen 12 und 14 Jahren alt. Zwischen den Einschätzungen aus Jugendlichen- und Elternsicht zeigen sich mittlere bis starke Zusammenhänge, in den Mittelwerten allerdings auch signifikante Unterschiede. So werden die verschiedenen Strategien aus Erziehendenperspektive häufiger angewandt als aus Sicht der Jugendlichen. Längsschnittlich nehmen die Mittelwerte der eingesetzten Strategien aus beiden Sichtweisen signifikant ab. Anders gestaltet es sich in Bezug auf die inkonsistente Mediation: Eltern schätzen sich insgesamt konsequenter in ihrer Medienerziehung ein als die Heranwachsenden dies tun und es handelt sich aus beiden Perspektiven um einen zeitlich relativ stabilen Aspekt.
\end{abstract}


How Does Media Education Change Over the Course of a Family's Biography? A Quantitative Longitudinal Analysis on the Stability of Parental Media Education from the Perspective of Parents and Adolescents

\begin{abstract}
Research has hardly taken a longer-term view on parental mediation into account yet. The aim of this paper is to investigate empirically parental mediation strategies (active, restrictive and technical mediation, as well as monitoring) over a long-term period. A multi-perspective approach allows the comparison between parental and adolescent perspectives. The question of how consistently parents behave in these educational strategies and whether one can observe changes over time plays a special role. The quantitative panel study «Trajectories of excessive Internet use in families (VEIF)» provides data from parental and adolescent perspectives over four waves at intervals of about one year. The paper gains a multi-perspective and longitudinal insight. The adolescents were between 12 and 14 years old at baseline. There are moderate to strong correlations between the assessments from adolescent and parental perspectives, but there are also significant differences in the mean values. The various strategies are used more frequently from the educator's perspective than from the adolescent perspective. Longitudinally, the mean values of the strategies decrease significantly from both perspectives. The situation is different regarding inconsistent mediation: parents rate themselves more consistent in their media education than do the adolescents, and this is a relatively stable aspect over time from both perspectives.
\end{abstract}

\title{
1. Medienerziehung als Voraussetzung für die Teilhabe an einer tiefgreifend mediatisierten Gesellschaft
}

Kinder und Jugendliche wachsen heute in einer Gesellschaft auf, deren Kommunikation grundlegend durch Medien geprägt ist. Hepp charakterisiert diese tiefgreifend mediatisierte Lebenswelt (Hepp 2020) anhand der folgenden fünf Merkmale: Ausdifferenzierung der Medientechnologie, deren wachsende Konnektivität, die Omnipräsenz digitaler (Mobil-)Kommunikation, eine beschleunigte Innovationsdichte und die Datafizierung allen Medienhandelns (Hepp 2018, 7). Das Aufwachsen von Kindern ist durch das Ausmass tiefgreifender Mediatisierung von Beginn an medial (mit-)geprägt (Krotz 2017) und digitale Medien sind aus dem Alltag von Kindern und Jugendlichen nicht mehr wegzudenken. Diese dienen nicht nur der Unterhaltung, sondern werden auch als Möglichkeit der Bildung, des Lernens, der gesellschaftlichen Teilhabe und Befähigung betrachtet (Hasebrink, Lampert, und Thiel 2019). Eine Auseinandersetzung mit digitalen Medien erscheint für Heranwachsende ebenso essenziell, wie das Herausbilden entsprechender Kompetenzen im Umgang mit diesen (Wagner, Gebel, 
und Lampert 2013), um die Heranwachsenden «zu einem sachgerechten, selbstbestimmten, kreativen und sozialverantwortlichen (sic!) Handeln in einer von Medien mitgestalteten Welt» (Tulodziecki 2017, 238) befähigen zu können. Die Corona-Pandemie und das damit einhergehende Distance Schooling stellten Familien vor zusätzliche Herausforderungen und verdeutlichen die Notwendigkeit medienbezogener Kompetenzen, sowohl von Kindern als auch Erwachsenen (Autorengruppe Bildungsberichterstattung 2020; Wößmann et al. 2021).

Die Familie bietet als soziale Domäne, in die das Kind hineingeboren wird, den Heranwachsenden die Möglichkeit für erste Medienerfahrungen (Kammerl und Kramer 2016). Eltern ermöglichen ihren Kindern durch das jeweilige Medienensemble der Familie den Zugang zu einem individuellen Medienrepertoire ${ }^{1}$, welches die Erziehenden andererseits auf bestimmte Medienpraktiken beschränken und in welchem sie - ebenso wie Geschwisterkinder - als Co-Nutzende oder Vorbilder auftreten (Çankaya und Odabaşı 2009; Eggert und Wagner 2016; Kammerl, Zieglmeier, und Wartberg 2020). Mit zunehmendem Alter erweitert sich das individuelle Medienrepertoire der Heranwachsenden, da diese selbst über ihre Mediennutzung entscheiden und der Einfluss anderer sozialer Domänen, wie Peers und Schule steigt. Nichtsdestotrotz erscheint die Familie immer noch als wichtiger Akteur für die jugendliche Mediennutzung (Kamin und Meister 2020). So bleiben Mediennutzungsmuster, die sich Heranwachsende in der frühen Kindheit angeeignet haben, häufig über einen längeren Zeitraum stabil (Paus-Hasebrink, Kulterer, und Sinner 2019) und ein starker familialer Einfluss auf die individuelle Mediennutzung ist auch im Jugendalter noch vorherrschend (Hasebrink, Lampert, und Thiel 2019).

Während also für mediensozialisationsbezogene Einflüsse schon Befunde vorliegen, gibt es bisher nur wenige Einblicke in die längerfristige Betrachtung aus medienerzieherischer Perspektive im Speziellen. Denn die grundlegende Annahme medienerzieherischer Theorien besteht darin, dass medienbezogene Sozialisation von Heranwachsenden mit Prozessen, die eine pädagogische Relevanz aufweisen, einhergehen (Iske 2012) und somit eine eigenständige Betrachtung dieser Prozesse nötig ist. So bemühen sich Eltern die Medienpraktiken der Heranwachsenden zu steuern und stehen vor der Anforderung ihr Medienerziehungsverhalten zugleich auf die neuen Herausforderungen und Chancen in einer tiefgreifend mediatisierten Gesellschaft abstimmen zu müssen (Schorb und Wagner 2013). Als wesentliche Voraussetzung für eine im Alltag funktionierende Medienerziehung nehmen Eltern dabei das Treffen klarer Vereinbarungen mit den Kindern und Jugendlichen wahr, die dann auch so umgesetzt werden (Wagner, Eggert, und Schubert 2016). Studien belegen jedoch auch, dass aufgestellte Regeln zur Mediennutzung in der Praxis nicht immer eingehalten werden (Feierabend, Plankenhorn, und Rathgeb 2017; Wagner, Eggert, und Schubert 2016).

1 Das Medienrepertoire umfasst alle Medienangebote, die das Kind regelmässig in seine Nutzungspraktiken mit einbezieht (Hasebrink und Hölig 2017). 
Elterliche Medienerziehung erscheint somit in einer tiefgreifend mediatisierten Gesellschaft als grundlegendes Element über das gesamte Kindes- und Jugendalter hinweg, um Heranwachsenden notwendige Kompetenzen zur sozialen Teilhabe vermitteln zu können. Wie sich die Entwicklung und Anpassung der Strategien über die Zeit hinweg gestalten, ist dabei noch ebenso wenig erforscht wie die praktische Umsetzung aufgestellter Regeln und Diskrepanzen in den Wahrnehmungen von Erziehenden und Heranwachsenden. Der vorliegende Beitrag versucht deshalb anhand von Daten der quantitativen Längsschnittstudie «Verläufe exzessiver Internetnutzung in Familien (VEIF)» (im Folgenden kurz: VEIF)² neue Einblicke auf diesem Gebiet zu liefern.

\section{Elterliche Medienerziehung und Parental Mediation als Systematisierung der eingesetzten Strategien}

Der Medienerziehungsbegriff ist im medienpädagogischen Diskurs aufgrund der Verknüpfung mit spezifischen Zielsetzungen und Wertvorstellungen grundsätzlich normativ aufgeladen (Spanhel 2006, 185). Die Aufgaben der Medienerziehung gestalten sich dabei grundsätzlich in zwei Bereichen, einerseits werden aus einer pädagogischen Zielsetzung heraus den Heranwachsenden Grenzen im Umgang mit Medien gesetzt und andererseits sollen die Kinder und Jugendlichen «innerhalb dieser Grenzen mit vielfältigen anregungs- und abwechslungsreichen medialen Umwelten konfrontiert werden» (Spanhel 2015, 13). Die praktische Umsetzung dieser medienerzieherischen Bemühungen ist dabei von konzeptionellen Sichtweisen auf medienbezogene Erziehungs- und Bildungsaufgaben abhängig, die je nach gesellschaftlichen Rahmenbedingungen historisch und individuell variieren (Tulodziecki, Herzig, und Grafe 2019, 162ff). So setzen Eltern in einer tiefgreifend mediatisierten Gesellschaft unterschiedlichste erzieherische Massnahmen ein, um ihre Kinder beim Erwerb eines verantwortungsvollen Umgangs mit digitalen Medien zu unterstützen (Mutlu-Bayraktar, Yılmaz, und Inan-Kaya 2018). Beispiele hierfür sind medienbezogene Gespräche zwischen Eltern und Kindern, eine gemeinsame Nutzung, das Kind zu einer selbständigen Erprobung digitaler Medien zu ermutigen, Erfahrungen auszutauschen, auf mögliche Zweifel oder Probleme der Heranwachsenden einzugehen, sich aktiv an Diskussionen zu beteiligen oder die Medienpraktiken der Heranwachsenden zu überwachen (einen systematischen Überblick geben Wahyuningrum, Suryanto, und Suminar 2020). Diese vielfältigen Handlungen lassen sich besonders schlüssig anhand des Konzepts der Parental Mediation systematisieren.

2 Das Forschungsprojekt «Verläufe exzessiver Internetnutzung in Familien (VEIF)» wird von Rudolf Kammerl (Friedrich-Alexander-Universität Erlangen-Nürnberg) und Lutz Wartberg (MSH Medical School Hamburg University of Applied Sciences and Medical University) geleitet und von der DFG gefördert. Weitere Informationen und bisherige Publikationen unter: https://www.medpaed.phil.fau.de/forschung/veif/. 
Parental Mediation bezeichnet die Art, wie Eltern versuchen die Mediennutzung ihres Kindes zu beeinflussen (Blum-Ross und Livingstone 2016) und stellt international seit den 1990er Jahren den einflussreichsten Ansatz zur Konzeptualisierung dar. Dabei wurden zunächst drei Strategien ausdifferenziert: aktive Mediation, restriktive Mediation und Co-Viewing (Blum-Ross und Livingstone 2016) oder Co-Using (Nathanson 1999; Valkenburg et al. 1999). Aktive Mediation meint eine bewusste Auseinandersetzung mit der Mediennutzung der Jugendlichen und eine Erklärung konsumierter Medieninhalte. Bei der restriktiven Mediation geht es demgegenüber um eine konkrete Regelsetzung bezüglich der Nutzungszeiten oder bestimmter Anwendungen bzw. Inhalte. Eine gemeinsame Mediennutzung von Eltern und Kindern wird als Co-Viewing oder Co-Using bezeichnet. Die Strategien der Parental Mediation wurden ursprünglich mit Fokus auf das Fernsehen entwickelt, jedoch im Zuge der fortschreitenden Mediatisierung angepasst und durch die Ausprägungen des Monitorings, also der Überprüfung besuchter Websites, genutzter Apps etc., bzw. der technischen Mediation ergänzt (Livingstone und Helsper 2008; Sonck, Nikken, und Haan 2013; Schaan und Melzer 2015; Pfetsch 2018). Die verschiedenen medienerzieherischen Handlungen, die mit dem Ansatz der Parental Mediation beschrieben werden können, existieren dabei nicht unabhängig voneinander, sondern werden auf unterschiedliche Weise von den Eltern eingesetzt und teils kombiniert (Blum-Ross und Livingstone 2016).

Da sich elterliche Medienerziehung nicht immer konsequent gestaltet, sondern Entscheidungen häufig situativ getroffen und entsprechende Regelungen nicht immer eingehalten werden (Kammerl et al. 2012), schlagen Kammerl, Zieglmeier und Wartberg (2020) vor, die genannten Strategien durch den Aspekt inkonsistenter Mediation zu erweitern. Diese wurde als Qualitätsmerkmal konzeptualisiert, um zu beschreiben, inwiefern Eltern bei ihren Versuchen, die kindliche Mediennutzung zu beeinflussen, im Alltag konsequente bzw. inkonsistente Verhaltensweisen zeigen.

\section{Ausgewählte Befunde zum Einfluss elterlicher Medienerziehungsstrategien}

Ein Einfluss der verschiedenen Strategien der Parental Mediation auf die kindliche Mediennutzung konnte bereits in zahlreichen empirischen Studien nachgewiesen werden (einen Überblick geben Pfetsch 2018; Wahyuningrum, Suryanto, und Suminar 2020). So berichten beispielsweise Fu et al. (2020) aktive Mediation als Prädiktor für das kindliche Mediennutzungsverhalten und deren Einstellung bezüglich Medien. Andererseits konnten Cabello-Hutt, Cabello und Claro (2018) statistisch belegen, dass weniger elterliche Medienerziehung mit höheren Onlinerisiken und mehr aktive Mediation sowie Co-Using mit höheren Chancen der Internetnutzung in Verbindung stehen. Eine stärkere restriktive Mediation wird andererseits mit geringeren OnlineRisiken, aber auch weniger Chancen in Zusammenhang gebracht. Eine Reihe von 
Studien berichtet zudem einen Einfluss der Parental Mediation auf exzessive Internetnutzung (Kammerl, Zieglmeier, und Wartberg 2020; Kalmus, Blinka, und Ólafsson 2015; Chng et al. 2015).

Der Einfluss einer (in)konsistenten Umsetzung der Parental Mediation Strategien ist bislang noch wenig erforscht. Die EXIF-Studie stellte zum ersten Mal einen Zusammenhang zwischen einem inkonsistenten Medienerziehungsverhalten und der exzessiven Mediennutzung her (Kammerl et al. 2012, 85ff). Zudem berichtet die FIM-Studie von einer Einhaltung medienbezogener Regeln je nach Anwendungsgebiet lediglich in rund der Hälfte bis drei Viertel der befragten Familien (Feierabend, Plankenhorn, und Rathgeb 2017, 18). Einen Zusammenhang mit einem problematischen Internetnutzungsverhalten konnten Kammerl, Zieglmeier und Wartberg (2020) auch statistisch belegen: neben anderen einflussreichen Faktoren der Medienerziehung und familialer Aspekte wurde dort für eine konsequentere Umsetzung medienerzieherischer Praktiken auch ein weniger problematisches Internetnutzungsverhalten zu einem späteren Messzeitpunkt berichtet.

Während inkonsistente Medienerziehung im Speziellen bislang noch wenig Beachtung im medienpädagogischen Diskurs findet, konnte bereits ein Einfluss inkonsistenter Erziehungsstrategien im Allgemeinen auf gestörte kindliche Verhaltensweisen nachgewiesen werden (Shelton, Frick, und Wootton 1996). Unter diesen Vorannahmen scheint ein positiver Einfluss konstanter medienbezogener Regelsetzungen auf eine selbstbestimmte und verantwortungsvolle Mediennutzung von Jugendlichen jedoch naheliegend.

\subsection{Unterschiede in der Wahrnehmung elterlicher Medienerziehung aus Sicht von Eltern und Heranwachsenden}

Während in den meisten Studien nur Eltern oder gar Expertinnen bzw. Experten in Bezug auf Medienerziehung zu Wort kommen, so scheint es doch essenziell, dass auch die Sichtweise der Heranwachsenden berücksichtigt wird. Ein multiperspektivischer Erhebungsansatz, der sowohl die Sicht von Erziehenden als auch der Jugendlichen einbezieht, ist hierbei von besonderem Wert. So zeigt beispielsweise Peled (2018), dass Eltern und Heranwachsende die eingesetzten Parental Mediation Strategien unterschiedlich wahrnehmen können. Aus der kindlichen Perspektive heraus werden dabei weniger medienerzieherische Praktiken wahrgenommen als aus Sicht der Eltern selbst. Zudem haben Eltern das Gefühl die Onlineaktivitäten ihres Kindes zu überblicken, wohingegen die Heranwachsenden diese Sichtweise nicht unbedingt teilen.

Bis auf diesen Einzelfall liegen allerdings noch kaum Erkenntnisse zu Unterschieden in der Wahrnehmung elterlicher Medienerziehung zwischen den Erziehenden und den zu Erziehenden vor und dementsprechende Erklärungen für die Diskrepanzen stehen noch aus. 


\subsection{Veränderungen elterlicher Medienerziehung im zeitlichen Verlauf}

Wie einleitend bereits hervorgehoben, passen Eltern ihre Medienerziehungsstrategien an die individuelle Mediennutzung und den Entwicklungsstand der Heranwachsenden an. So kommen bei älteren Kindern und Jugendlichen insgesamt weniger Parental Mediation Strategien zum Einsatz, als bei jüngeren (Kerr, Stattin, und Burk 2010; Peled 2018; Dedkova und Smahel 2020). Cabello-Hutt, Cabello und Claro (2018) berichten, dass mit zunehmendem Alter Co-Using und aktive Mediation abnehmen, wohingegen Lee (2013) diese Abnahme im Bereich der restriktiven Mediation sieht.

Symons et al. (2017) leiten aus ihren qualitativen Ergebnissen ab, Parental Mediation als dynamischen Prozess zu betrachten, der durch die täglichen Interaktionen von Jugendlichen und Eltern hervorgebracht wird. So ist es auch vorstellbar, dass durch neue mediale Entwicklungen, wie beispielsweise die beinahe selbstverständliche Omnipräsenz des Smartphones seit einigen Jahren, Handlungsweisen hinzukommen oder durch den im Sozialisationsprozess abnehmenden Einfluss von Eltern andere Praktiken wegfallen bzw. abnehmen.

Längsschnittliche Betrachtungen elterlicher Medienerziehung kommen bisher zu unterschiedlichen Schlüssen. So konnte die Studie von Nikken und de Graaf (2013), die Jugendliche zu zwei Zeitpunkten im Abstand von etwa einem Jahr befragte, keinen statistisch signifikanten Zusammenhang zwischen aktiver bzw. restriktiver Mediation zwischen den beiden Erhebungszeitpunkten belegen. Dementgegen steht die Studie von Padilla-Walker et al. (2012): hier konnte über zwei Jahre ein recht stabiler Zusammenhang restriktiver, sowie aktiver Medienerziehung über drei Erhebungszeitpunkte hinweg festgestellt werden. Beide längsschnittliche Beispiele lassen erkennen, dass auch hier bisher nur ein sehr kurzer Zeitraum des Aufwachsens abgedeckt werden konnte und noch uneinheitliche Ergebnisse vorliegen.

Der vorliegende Beitrag versucht von dem dargestellten Forschungsstand ausgehend neue Erkenntnisse in zwei Richtungen zu liefern. So sollen einerseits Diskrepanzen in der Wahrnehmung medienerzieherischer Praktiken aus Sicht von Erziehenden und Heranwachsenden genauer beleuchtet werden. Andererseits findet eine längsschnittliche Betrachtung der Parental Mediation Strategien ebenso wie deren konsequente Umsetzung in der Analyse Beachtung. Daraus ergeben sich die folgenden Forschungsfragen:

- FF1: Welche Zusammenhänge bzw. Unterschiede zeigen sich zwischen der Einschätzung der Medienerziehungsstrategien seitens der Heranwachsenden und der Erziehenden zum jeweiligen Erhebungszeitpunkt?

- FF2: Inwiefern verändert sich die Einschätzung der Medienerziehung aus Sicht der Erziehenden über die Zeit hinweg?

- FF3: Inwiefern verändert sich die Einschätzung der Medienerziehung aus Sicht der Jugendlichen über die Zeit hinweg? 


\section{Methodisches Vorgehen und Sampling}

Um diese Fragen zu beantworten, wurden die Daten der quantitativen Längsschnittstudie VEIF analysiert. Grundlage bildeten vier Erhebungswellen, bei denen Jugendliche und jeweils ein Haupterziehender befragt wurden $\left(N_{2016}=1095 ; N_{2017}=958\right.$; $N_{2018}=852 ; N_{2019}=633$ Dyaden). Zum ersten Erhebungszeitpunkt waren die Heranwachsenden zwischen 12 und 14 Jahre alt und die Befragungen fanden im Abstand von etwa einem Jahr statt. Durch die theoretische Anlage und die Rekrutierungsstrategie der Studie sind Jugendliche mit einem problematischen Internetnutzungsverhalten stark überrepräsentiert (der Anteil liegt zu allen Erhebungszeitpunkten bei rund $70 \%)$.

Teil des Fragebogens waren auch fünf Skalen zur elterlichen Medienerziehung, die aus beiden Sichtweisen beantwortet wurden. Die Subskalen zur aktiven Mediation, restriktiven Mediation, des Monitorings und der technischen Mediation wurden dabei aus dem EU-Kids Online Project übernommen (Dürager und Sonck 2014). Die Subskala zur aktiven Mediation umfasst beispielsweise gemeinsame Aktivitäten im Internet oder Empfehlungen für Aktivitäten durch die Eltern. Sie besteht aus fünf Items in einem dichotomen Antwortformat $(0=$ «nein», $1=$ «ja»). Mit der Skala zur restriktiven Mediation, bestehend aus fünf Items in dreistufigem Antwortformat $(0=$ «erlaubt, wann immer es will», $1=$ «erlaubt nach eingeholter Erlaubnis oder Aufsicht», 2 = «nie erlaubt»), werden vorwiegend Verbote bezüglich der jugendlichen Internetnutzung erfasst. Die Subskala des Monitorings umfasst vier verschiedene Items, die insbesondere die elterliche Kontrolle der Social Media Nutzung durch den Jugendlichen fokussieren. Diese waren ebenfalls in einem dichotomen Antwortformat $\left(0=\right.$ «nein», $1=$ «ja») gestellt. ${ }^{3}$ Die Subskala zur technischen Mediation setzt sich aus drei Items zusammen, ebenfalls in einem dichotomen Antwortformat ( $0=$ «nein», $1=$ «ja»). Die Aussagen beziehen sich vorwiegend auf den Einsatz von Software zur zeitlichen oder inhaltlichen Beschränkung der jugendlichen Internetnutzung. ${ }^{4}$ Eine Skala zum inkonsistenten Medienerziehungsverhalten, die erfassen soll wie häufig die Erziehenden von festgelegten Regeln abweichen, wurde zusätzlich als Qualitätsmerkmal elterlicher Medienerziehung ergänzt (Kammerl, Zieglmeier, und Wartberg 2020). Diese besteht aus zwei Items in fünfstufigen Antwortformat ( $0=$ «fast nie», $1=$ «manchmal», $2=$ «oft», $3=$ «fast immer», sowie $4=$ «lch gebe keine Zeit vor» aus Elternsicht und $4=$ «Sie geben keine Zeit vor»/«Sie verbieten keine Internetangebote» aus Jugendlichensicht). ${ }^{5}$

3 Eine zusätzliche Antwortmöglichkeit (für die Eltern «nicht zutreffend/Kind nutzt das nicht» und für die Jugendlichen «weiß ich nicht») wurde als fehlender Wert nicht mit in weitere Berechnungen einbezogen.

4 Auch hier stand für die Heranwachsenden eine dritte Antwortoption ( 2 = «weiß ich nicht») zur Verfügung, deren Wahl nicht weiter einbezogen wurde.

5 Für weitere Analysen wurden nur die Antwortmöglichkeiten 0 bis 3 einbezogen und die letzte Antwortoption als fehlender Wert betrachtet. 
Für alle fünf Subskalen wurde jeweils für die Einschätzung der Jugendlichen und der Eltern die Reliabilität der Skala anhand von Cronbachs Alpha berechnet (Tab. 1). Kritisch anzumerken sind an dieser Stelle die eher niedrigen Reliabilitäten der Subskala der aktiven Mediation (zwischen minimal .51 und maximal .64). Eine Überprüfung des Weglassens einzelner Items aus der Skala führt an dieser Stelle allerdings nicht zu einer höheren Reliabilität. Dabei scheint es sich um keinen spezifischen Stichprobeneffekt zu handeln, sondern auch Dürager und Sonck berichten von niedrigeren Reliabilitäten für die deutsche Übersetzung des eingesetzten Instruments zur Erfassung der aktiven Meditation im Gegensatz zu anderen Sprachen (Dürager und Sonck 2014, 13). Trotz der eher schwachen Reliabilität wurde sich in diesem Beitrag dafür entschieden dennoch Ergebnisse für das gesamte Instrument und damit auch aktive Mediation zu berichten. Die Analysen müssen allerdings mit einem kritischen Auge betrachtet werden.

\begin{tabular}{|l|c|c|c|c|c|}
\hline & & 1. EW & 2. EW & 3. EW & 4. EW \\
\hline Aktive Mediation & ES & .55 & .51 & .64 & .58 \\
& JS & .57 & .56 & .64 & .61 \\
\hline Restriktive Mediation & ES & .68 & .72 & .75 & .76 \\
& JS & .70 & .74 & .78 & .79 \\
\hline Monitoring & ES & .76 & .78 & .79 & .79 \\
& JS & .78 & .82 & .82 & .83 \\
\hline Technische Mediation & ES & .65 & .73 & .68 & .69 \\
& JS & .68 & .73 & .78 & .77 \\
\hline Inkonsistentes Medienerziehungsverhalten & ES & .69 & .76 & .74 & .68 \\
& JS & .67 & .78 & .74 & .59 \\
\hline
\end{tabular}

Tab. 1.: Reliabilität der Subskalen zur Parental Mediation. Anmerkungen: Werte für Cronbachs Alpha jeweils für die Skalen aus ES = Elternsicht und JS = Jugendlichensicht über vier Erhebungswellen (EW).

Da durch das Oversampling im VEIF-Projekt Jugendliche mit einem problematischen Mediennutzungsverhalten überrepräsentiert sind, wurden Mittelwertunterschiede beider Gruppen anhand eines t-Tests für unabhängige Stichproben berechnet, um Effekte der Gruppenzugehörigkeit auf die Subskalen der Parental Mediation zu überprüfen (Tab. 2). Während sich bei einigen Subskalen Mittelwertunterschiede zeigen, lässt sich festhalten, dass die Unterschiede über die Erhebungszeiträume nicht konstant bleiben und bei einigen Subskalen keine signifikanten Unterschiede festgestellt werden können. Aus diesem Grund wird das Oversampling der Jugendlichen mit einem problematischen Internetnutzungsverhalten im Folgenden nicht weiter berücksichtigt.

Zudem wurde das arithmetische Mittel (Tab. 3) berechnet, wobei ein höherer Mittelwert für eine stärkere Ausprägung der Subskalen der aktiven Mediation, der restriktiven Mediation, des Monitorings und der technischen Mediation der jugendlichen 
Internetnutzung spricht. Bei der Skala der inkonsistenten Medienerziehung spricht ein höherer Mittelwert dafür, dass ein solches Verhalten häufiger wahrgenommen wird.

\begin{tabular}{|l|c|c|c|c|c|}
\hline \multicolumn{2}{|c}{} & 1. EW & 2. EW & 3. EW & 4. EW \\
\cline { 3 - 6 } & & T & T & T & T \\
\hline Aktive Mediation & ES & $2.40^{\star}$ & -.07 & $3.85^{\star \star}$ & $4.00^{\star \star}$ \\
& JS & $2.44^{\star}$ & .70 & $3.61^{\star \star}$ & $3.41^{\star \star}$ \\
\hline Restriktive Mediation & ES & $2.22^{\star}$ & $2.53^{\star}$ & $3.61^{\star \star}$ & $3.95^{\star \star}$ \\
& JS & $2.06^{\star}$ & 1.84 & $3.77^{\star \star}$ & $4.10^{\star \star}$ \\
\hline Monitoring & ES & -.01 & .50 & .10 & 1.17 \\
& JS & -.94 & .29 & -.87 & 1.74 \\
\hline Technische Mediation & ES & .66 & 1.01 & 1.44 & $2.58^{\star}$ \\
& JS & .79 & 1.59 & 1.85 & $2.16^{\star}$ \\
\hline Inkonsistentes Medienerziehungsverhalten & ES & $-9.72^{\star \star}$ & $-5.05^{\star \star}$ & -1.66 & -.45 \\
& JS & $-7.99^{\star \star}$ & $-4.15^{\star \star}$ & $-4.48^{\star \star}$ & -1.67 \\
\hline
\end{tabular}

Tab. 2.: Mittelwertunterschiede zwischen Test- und Kontrollgruppe in den Subskalen der Parental Mediation. Anmerkungen: $\mathrm{ES}=$ Elternsicht, JS = Jugendlichensicht; ${ }^{\star} \mathrm{p}<.05,{ }^{\star \star} \mathrm{p}<.01$.

Zur Analyse der aufgeworfenen Forschungsfragen wurde die Statistiksoftware SPSS 26.0 (IBM 2019, New York, USA) genutzt. Berechnet wurden zum jeweiligen Erhebungszeitpunkt Mittelwerte, Standardabweichungen, Reliabilitätskoeffizienten, Korrelationen und Mittelwertunterschiede zwischen den Subskalen aus Sicht von Eltern und Heranwachsenden. Um eine möglichst valide Aussage zur Entwicklung der Parental Mediation zu erhalten, wurde für die längsschnittlichen Analysen ein listenweiser Fallausschluss verwendet (N2019 =633). Zudem wurde eine einfaktorielle Varianzanalyse mit Messwiederholung berechnet, um zu überprüfen, ob sich die Mittelwerte zu den verschiedenen Erhebungszeitpunkten unterscheiden und wie sich ein solcher Verlauf beschreiben lässt.

\section{Ergebnisse}

Im Folgenden werden die Ergebnisse anhand der oben hergeleiteten Forschungsfragen strukturiert erläutert. Während sich der erste Teil den Zusammenhängen bzw. Unterschieden zwischen den Subskalen der Parental Mediation aus Eltern- und Jugendlichensicht widmet, beschäftigen sich die beiden darauffolgenden Abschnitte mit den längsschnittlichen Analysen aus beiden Perspektiven. 


\subsection{Zusammenhang zwischen der Einschätzung aus Eltern- und Jugendlichenperspektive}

Im Vergleich der beiden Perspektiven zeigen sich seitens der elterlichen Einschätzung der verschiedenen Parental Mediation Strategien leicht höhere Mittelwerte zu allen Erhebungszeitpunkten in den Subskalen der aktiven, restriktiven, technischen Mediation und des Monitorings. Gegenteilig zeigt sich dies bei der inkonsistenten Mediation (Tab. 3). Diese Mittelwertunterschiede bestätigen die t-Tests für abhängige Stichproben, die für alle Subskalen und Erhebungen - mit Ausnahme der technischen Mediation zum dritten und vierten Erhebungszeitpunkt und der inkonsistenten Medienerziehung zum vierten Messzeitpunkt - signifikant sind (Tab. 4). Darüber hinaus zeigt sich über alle vier Erhebungswellen hinweg ein mittlerer bis starker signifikanter Zusammenhang zwischen Einschätzungen von Erziehenden und Heranwachsenden (Tab. 3).

\begin{tabular}{|c|c|c|c|c|c|}
\hline & $\begin{array}{c}\mathrm{M} \\
(\mathrm{ES})\end{array}$ & $\begin{array}{c}\text { SD } \\
\text { (ES) }\end{array}$ & $\begin{array}{c}M \\
\text { (JS) }\end{array}$ & $\begin{array}{c}\text { SD } \\
\text { (JS) }\end{array}$ & $\begin{array}{l}\text { Pear- } \\
\text { son } \\
\text { Korre- } \\
\text { lation }\end{array}$ \\
\hline Aktive Mediation (1. EW) & .59 & .27 & .50 & .28 & $.62^{\star *}$ \\
\hline Aktive Mediation (2. EW) & .56 & .27 & .49 & .28 & $.60^{\star *}$ \\
\hline Aktive Mediation (3. EW) & .49 & .29 & .42 & .28 & $.68^{\star *}$ \\
\hline Aktive Mediation (4. EW) & .45 & .26 & .38 & .26 & $.50^{\star \star}$ \\
\hline Restriktive Mediation (1. EW) & .59 & .29 & .56 & .30 & $.78^{\star \star}$ \\
\hline Restriktive Mediation (2. EW) & .53 & .31 & .49 & .32 & $.77^{\star \star}$ \\
\hline Restriktive Mediation (3. EW) & .42 & .32 & .38 & .33 & $.82^{\star \star}$ \\
\hline Restriktive Mediation (4. EW) & .32 & .32 & .30 & .32 & $.78^{\star *}$ \\
\hline Monitoring (1. EW) & .39 & .38 & .31 & .37 & $.57^{\star *}$ \\
\hline Monitoring (2. EW) & .36 & .37 & .29 & .37 & $.58^{\star \star}$ \\
\hline Monitoring (3. EW) & .31 & .37 & .23 & .34 & $.62^{* *}$ \\
\hline Monitoring (4. EW) & .25 & .34 & .19 & .32 & $.61^{\star *}$ \\
\hline Technische Mediation (1. EW) & .22 & .31 & .21 & .34 & $.67^{\star \star}$ \\
\hline Technische Mediation (2. EW) & .20 & .32 & .18 & .32 & $.67^{* *}$ \\
\hline Technische Mediation (3. EW) & .13 & .26 & .14 & .30 & $.72^{\star *}$ \\
\hline Technische Mediation (4. EW) & .11 & .25 & .10 & .25 & $.71^{\star \star}$ \\
\hline Inkonsistentes Medienerziehungsverhalten (1. EW) & 1.86 & .71 & 1.90 & .73 & $.59^{\star *}$ \\
\hline Inkonsistentes Medienerziehungsverhalten (2. EW) & 1.85 & .68 & 1.93 & .74 & $.62^{\star *}$ \\
\hline Inkonsistentes Medienerziehungsverhalten (3. EW) & 1.84 & .72 & 1.93 & .71 & $.57^{\star \star}$ \\
\hline Inkonsistentes Medienerziehungsverhalten (4. EW) & 1.79 & .66 & 1.82 & .65 & $.48^{\star \star}$ \\
\hline
\end{tabular}

Tab. 3.: Mittelwerte, Standardabweichungen und Korrelationen für die Subskalen der Parental Mediation aus Elternsicht. Anmerkungen: ${ }^{*} p<.05,{ }^{\star *} p<.01$ über vier Erhebungswellen (EW). 


\begin{tabular}{|l|c|c|c|c|}
\hline & 1. EW & 2. EW & 3. EW & 4. EW \\
\hline & T & T & T & T \\
\hline Aktive Mediation & $12.70^{\star \star}$ & $9.23^{\star \star}$ & $9.22^{\star \star}$ & $7.66^{\star \star}$ \\
\hline Restriktive Mediation & $5.27^{\star \star}$ & $5.79^{\star \star}$ & $6.60^{\star \star}$ & $3.23^{\star \star}$ \\
\hline Monitoring & $8.17^{\star \star}$ & $5.45^{\star \star}$ & $7.98^{\star \star}$ & $4.53^{\star \star}$ \\
\hline Technische Mediation & $2.33^{\star}$ & $2.85^{\star \star}$ & -1.59 & 1.64 \\
\hline Inkonsistentes Medienerziehungsverhalten & $-2.12^{\star}$ & $-3.36^{\star \star}$ & $-3.05^{\star \star}$ & -1.53 \\
\hline
\end{tabular}

Tab. 4.: Mittelwertunterschiede zwischen Eltern- und Jugendlichenperspektive in den Subskalen der Parental Mediation. Anmerkungen: ES = Elternsicht, JS = Jugendlichensicht; ${ }^{\star} \mathrm{p}<.05,{ }^{* *} \mathrm{p}<.01$.

\subsection{Entwicklung der Parental Mediation aus Elternperspektive}

Bei der Betrachtung der aktiven, restriktiven und technischen Mediation, ebenso wie dem Monitoring und der inkonsistenten Medienerziehung über vier Erhebungswellen aus Elternsicht (Tab. 5) fällt zunächst auf, dass über alle Subskalen hinweg die Mittelwerte geringfügig, aber konstant abnehmen. Zudem zeigen sich zwischen allen Subskalen über die vier Erhebungswellen hinweg positive, signifikante Korrelationen. Die Stärke variiert zwischen minimal .24 und maximal .48, was einem mittleren Zusammenhang zwischen den Erhebungszeitpunkten entspricht.

Eine Varianzanalyse mit Messwiederholung (Sphärizität angenommen: Mauchly$W(5)=.986, p=.110)$ zeigt, dass die aktive Mediation der Eltern sich über die vier Erhebungswellen hinweg statistisch signifikant unterscheiden $(F(3)=70.10, p<.001$, partielles $\eta 2=.100$ ). Für die restriktive Mediation zeigt die Varianzanalyse mit Messwiederholung mit Greenhouse-Geisser-Korrektur, dass diese sich in den Erhebungswellen statistisch signifikant unterscheiden $(F(2,96)=143.21, p<.001$, partielles $\eta^{2}=.185$ ). Die Analyse des Monitorings (Sphärizität angenommen: Mauchly$W(5)=.992, p=.391$ ) zeigt ebenfalls einen signifikanten Unterschied zwischen den Erhebungszeitpunkten $\left(F(2.98)=31.52, p<.001\right.$, partielles $\left.\eta^{2}=.048\right)$. Für die technische Mediation kann nach einer Greenhouse-Geisser-Korrektur der Varianzanalyse mit Messwiederholung ebenfalls ein statistisch signifikanter Unterschied berichtet werden $\left(F(2,82)=38.76, p<.001\right.$, partielles $\left.\eta^{2}=.058\right)$. Für die Skala der inkonsistenten Medienerziehung gab es hingegen keinen statistisch signifikanten Unterschied zwischen den Messzeitpunkten $(F(3)=3.31, p=.020)$. Diese Ergebnisse verdeutlichen, dass alle Parental Mediation Strategien im Verlauf von vier Jahren statistisch weniger häufig angewandt werden. Anders gestaltet sich dies bei der Skala zur inkonsistenten Medienerziehung, die von Eltern über alle Erhebungszeitpunkte etwa gleich ausgeprägt wahrgenommen wird. Die grafische Aufbereitung (Abb. 1) verdeutlicht diese Ergebnisse. ${ }^{6}$

6 Zur besseren Übersichtlichkeit wurde auf die Darstellung der inkonsistenten Medienerziehung verzichtet. 


\begin{tabular}{|c|c|c|c|c|c|}
\hline & M & SD & 1. EW & 2. EW & 3. EW \\
\hline Aktive Mediation (1. EW) & .59 & .28 & - & & \\
\hline Aktive Mediation (2. EW) & .54 & .26 & $.39^{\star \star}$ & - & \\
\hline Aktive Mediation (3. EW) & .46 & .28 & $.45^{\star \star}$ & $.43^{\star \star}$ & - \\
\hline Aktive Mediation (4. EW) & .45 & .26 & $.36^{\star \star}$ & $.36^{\star \star}$ & $.49^{\star \star}$ \\
\hline Restriktive Mediation (1. EW) & .59 & .29 & - & & \\
\hline Restriktive Mediation (2. EW) & .52 & .32 & $.44^{\star \star}$ & - & \\
\hline Restriktive Mediation (3. EW) & .42 & .32 & $.40^{\star \star}$ & $.46^{\star \star}$ & - \\
\hline Restriktive Mediation (4. EW) & .32 & .32 & $.34^{\star \star}$ & $.37^{\star \star}$ & $.41^{\star \star}$ \\
\hline Monitoring (1. EW) & .41 & .38 & - & & \\
\hline Monitoring (2. EW) & .37 & .37 & $.30^{\star \star}$ & - & \\
\hline Monitoring (3. EW) & .30 & .36 & $.28^{\star \star}$ & $.33^{\star \star}$ & - \\
\hline Monitoring (4. EW) & .25 & .34 & $.25^{\star \star}$ & $.28^{\star \star}$ & $.29^{\star \star}$ \\
\hline Technische Mediation (1. EW) & .22 & .31 & - & & \\
\hline Technische Mediation (2. EW) & .21 & .32 & $.36^{\star \star}$ & - & \\
\hline Technische Mediation (3. EW) & .13 & .26 & $.40^{\star \star}$ & $.44^{\star \star}$ & - \\
\hline Technische Mediation (4. EW) & .11 & .25 & $.36^{\star \star}$ & $.44^{\star \star}$ & $.47^{\star \star}$ \\
\hline Inkonsistentes Medienerziehungsverhalten (1. EW) & 1.87 & .70 & - & & \\
\hline Inkonsistentes Medienerziehungsverhalten (2. EW) & 1.87 & .69 & $.42^{\star \star}$ & - & \\
\hline Inkonsistentes Medienerziehungsverhalten (3. EW) & 1.86 & 69 & $.32^{\star \star}$ & $48^{* *}$ & - \\
\hline Inkonsistentes Medienerziehungsverhalten (4. EW) & 1.76 & .64 & $.24^{\star \star}$ & $.29^{\star \star}$ & $.28^{\star *}$ \\
\hline
\end{tabular}

Tab. 5.: Mittelwerte, Standardabweichungen und Pearson Korrelationen für die Subskalen der Parental Mediation aus Elternsicht. Anmerkungen: ${ }^{\star} p<.05,{ }^{\star *} p<.01$ über vier Erhebungswellen (EW).

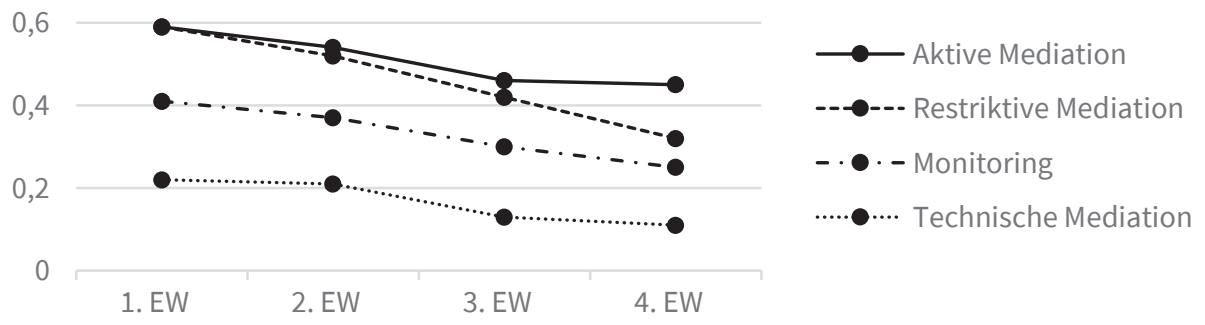

Abb. 1.: Mittelwerte der Parental Mediation aus Elternsicht über die vier Erhebungswellen (EW).

\subsection{Entwicklung der Parental Mediation aus Jugendlichenperspektive}

Die Analyse der Subskalen der Parental Mediation über vier Erhebungswellen aus Sicht der Jugendlichen (Tab. 6) liefert ein ähnliches Bild, wie die Betrachtung der Mittelwerte und Korrelationen aus Elternsicht. Die Mittelwerte der Skalen zur aktiven, restriktiven und technischen Mediation, ebenso wie des Monitorings nehmen 
im Laufe der Erhebungswellen stetig ab. Anders verhält es sich beim inkonsistenten Medienerziehungsverhalten, dessen Skalenmittelwert zum zweiten Erhebungszeitpunkt im Vergleich zur ersten Welle geringfügig höher ausfällt. Zudem zeigen sich zwischen allen Subskalen über die vier Erhebungswellen hinweg positive, signifikante Korrelationen. Die Stärke des Zusammenhangs variiert bei der Einschätzung der Jugendlichen etwas stärker zwischen minimal .22 und maximal .56, woraus sich ebenfalls moderate Korrelationen schliessen lassen.

\begin{tabular}{|l|c|c|c|c|c|}
\hline & $\mathrm{M}$ & $\mathrm{SD}$ & $1 . \mathrm{EW}$ & 2. EW & 3. EW \\
\hline Aktive Mediation (1. EW) & .49 & .28 & - & & \\
\hline Aktive Mediation (2. EW) & .47 & .26 & $.41^{\star \star}$ & - & \\
\hline Aktive Mediation (3. EW) & .39 & .27 & $.40^{\star \star}$ & $.47^{\star \star}$ & - \\
\hline Aktive Mediation (4. EW) & .38 & .26 & $.31^{\star \star}$ & $.43^{\star \star}$ & $.53^{\star \star}$ \\
\hline Restriktive Mediation (1. EW) & .56 & .29 & - & & \\
\hline Restriktive Mediation (2. EW) & .48 & .32 & $.44^{\star \star}$ & - & \\
\hline Restriktive Mediation (3. EW) & .38 & .32 & $.40^{\star \star}$ & $.46^{\star \star}$ & - \\
\hline Restriktive Mediation (4. EW) & .30 & .32 & $.34^{\star \star}$ & $.37^{\star \star}$ & $.41^{\star \star}$ \\
\hline Monitoring (1. EW) & .30 & .36 & - & & \\
\hline Monitoring (2. EW) & .30 & .36 & $.42^{\star \star}$ & - & \\
\hline Monitoring (3. EW) & .21 & .32 & $.34^{\star \star}$ & $.36^{\star \star}$ & - \\
\hline Monitoring (4. EW) & .19 & .32 & $.28^{\star \star}$ & $.22^{\star \star}$ & $.29^{\star *}$ \\
\hline Technische Mediation (1. EW) & .21 & .33 & - & & \\
\hline Technische Mediation (2. EW) & .19 & .32 & $.37^{\star \star}$ & - & \\
\hline Technische Mediation (3. EW) & .15 & .31 & $.39^{\star \star}$ & $.52^{\star \star}$ & - \\
\hline Technische Mediation (4. EW) & .10 & .26 & $.33^{\star \star}$ & $.49^{\star \star}$ & $.56^{\star \star}$ \\
\hline Inkonsistentes Medienerziehungsverhalten (1. EW) & 1.90 & .70 & - & & \\
\hline Inkonsistentes Medienerziehungsverhalten (2. EW) & 1.92 & .74 & $.35^{\star \star}$ & - & \\
\hline Inkonsistentes Medienerziehungsverhalten (3. EW) & 1.88 & .67 & $.29^{\star \star}$ & $40^{\star \star}$ & - \\
\hline Inkonsistentes Medienerziehungsverhalten (4. EW) & 1.81 & .66 & $.29^{\star \star}$ & $.40^{\star \star}$ & $.28^{\star \star}$ \\
\hline
\end{tabular}

Tab. 6.: Mittelwerte, Standardabweichungen und Pearson Korrelationen für die Subskalen der Parental Mediation aus Jugendlichensicht. Anmerkungen: ${ }^{*} p<.05,{ }^{\star *} p<.01$ über vier Erhebungswellen (EW).

Die Varianzanalyse mit Messwiederholung wurde auch für die Skalen der Parental Mediation aus Jugendlichensicht durchgeführt (Abb. 2). Hier wurde für die Subskalen der aktiven, restriktiven und technischen Mediation, ebenso wie des Monitorings eine Greenhouse-Geisser-Korrektur nötig. Daraufhin zeigt sich für die aktive Mediation ein statistisch signifikanter Unterschied zwischen den Messzeitpunkten $\left(F(2.88)=44.82, p<.001\right.$, partielles $\left.\eta^{2}=.066\right)$, ebenso wie bei der restriktiven Mediation $\left(F(2.96)=133.86, p<.001\right.$, partielles $\left.\eta^{2}=.175\right)$. Ein signifikanter Unterschied 
ergibt sich dann ebenfalls in den Mittelwerten des Monitorings $(F(2.95)=19.99$, $\mathrm{p}<.001$, partielles $\left.\eta^{2}=.040\right)$ und der technischen Mediation $(F(2.77)=17.95, p<.001$, partielles $\left.\eta^{2}=.041\right)$. Bei der Einschätzung der Jugendlichen zur inkonsistenten Medienerziehung konnte kein statistisch signifikanter Unterschied zwischen den Messzeitpunkten festgestellt werden $(F(3)=2.20, p=.087)$. Ebenso wie aus Elternsicht verdeutlicht sich, dass die Mittelwerte der Parental Mediation Subskalen statistisch signifikant abnehmen. Auch hier verhält es sich mit der inkonsistenten Medienerziehung ähnlich wie aus Elternperspektive dargestellt: es sind keine statistisch relevanten Effekte nachzuweisen.
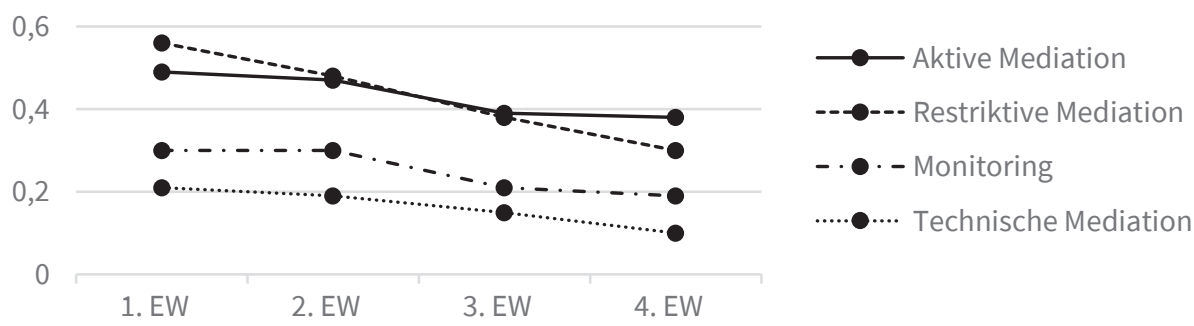

Abb. 2.: Mittelwerte der Parental Mediation aus Jugendlichensicht über die vier Erhebungswellen (EW).

\subsection{Zusammenfassung der Ergebnisse}

Im Querschnitt zeigen sich für alle vier Erhebungswellen signifikante mittlere bis starke Effektstärken zwischen der Einschätzung der aktiven, restriktiven und technischen Mediation, ebenso wie des Monitorings und des inkonsistenten Medienerziehungsverhaltens. Diese Zusammenhänge deuten grundsätzlich auf eine ähnliche Einschätzung seitens von Eltern und Jugendlichen zu allen Erhebungszeitpunkten hin. Dieser Einblick kann durch die Betrachtung der Mittelwerte und bestehende Mittelwertunterschiede erweitert werden. Bis auf die genannten Ausnahmen (s. oben) können zu allen Erhebungszeitpunkten signifikante Unterschiede zwischen beiden Perspektiven berichtet werden. So nehmen die Eltern durchschnittlich mehr eingesetzte Parental Mediation Strategien (aktive, restriktive und technische Mediation sowie Monitoring) wahr, als die Heranwachsenden. Eine gegenteilige Einschätzung zeigt sich hingegen bei der Wahrnehmung inkonsistenter Medienerziehung. So scheinen die Eltern ihre eigene Medienerziehung als konsequenter zu betrachten als ihre Jugendlichen.

In der längsschnittlichen Analyse zeigen sich bei allen Subskalen der aktiven, restriktiven und technischen Mediation sowie des Monitorings über die Zeit signifikant abnehmende Mittelwerte sowohl aus Eltern-, als auch aus Jugendlichensicht. Die mittleren Effektstärken der einzelnen Subskalen zwischen den vier Erhebungszeitpunkten deuten auf eine gleichmässige Abnahme der Einschätzungen hin. Eine signifikante Abnahme lässt sich andererseits nicht für das Qualitätsmerkmal der inkonsistenten Medienerziehung nachweisen. 


\section{Diskussion und Fazit}

Eine tiefgreifend mediatisierte Lebenswelt wirkt sich insbesondere auf Familien und deren Erziehungsverhalten aus, da unterschiedlichste digitale Medien bereits ab der frühen Kindheit allgegenwärtig im Leben der Heranwachsenden zu finden sind. Eltern nutzen verschiedene medienerzieherische Strategien, um ihre Kinder bei den neuen Herausforderungen, die sich daraus ergeben, zu unterstützen und diese bestmöglich zu fördern. Die Erziehenden formulieren dabei nicht nur restriktiv Regeln zur Mediennutzung, sondern setzen sich auch aktiv mit den Medienpraktiken ihrer Kinder auseinander, nehmen technische Einschränkungen an Geräten vor und überprüfen genutzte Inhalte und Anwendungen.

Die Ergebnisse der querschnittlichen Analysen verweisen darauf, dass sich die Einschätzungen der Heranwachsenden und der Erziehenden hinsichtlich der elterlichen Medienerziehung zumindest zum Teil decken. Jedoch schätzen die Eltern ihre eigenen medienerzieherischen Bestrebungen in den Bereichen der aktiven, restriktiven und technischen Medienerziehung und des Monitorings als wesentlich höher ein, als die Jugendlichen dies tun würden. Im Gegensatz dazu nehmen die Heranwachsenden die angewandten Erziehungsstrategien als weniger konsequent umgesetzt wahr als die befragten Elternteile. Worin diese Unterschiede begründet liegen, lässt sich an dieser Stelle bisher nur Mutmassen: Ob sie sich nur mit den individuellen Blickwinkeln der unterschiedlichen Akteurinnen und Akteure begründen lassen, die medienerzieherischen Massnahmen wirklich nicht - oder zumindest weniger als gedacht - bei den Jugendlichen ankommen oder die Elternteile aus sozialer Erwünschtheit heraus geantwortet haben, bleibt offen. Mögliche Begründungszusammenhänge müssten an dieser Stelle durch weitere empirische Befunde abgesichert werden. Jedoch lässt sich eindeutig festhalten, dass der multiperspektivischen Betrachtung medienerzieherischer Praktiken aus Sicht der Erziehenden und zu Erziehenden künftig mehr Bedeutung beigemessen werden sollte, um einen umfassenderen Einblick in den medienbezogenen Familienalltag und damit verbundene Aushandlungsprozesse zu erhalten.

Eine Abnahme der eingesetzten Strategien der aktiven, restriktiven und technischen Mediation sowie des Monitorings aus Eltern- und Jugendlichenperspektive zeigt sich mit Blick auf das Alter der jugendlichen Befragten und einer damit verbundenen zunehmenden Autonomie als logische Konsequenz. So nimmt der erzieherische Einfluss der Eltern im Zuge des Sozialisationsprozesses stetig ab und auch in Bezug auf die Mediennutzung werden zunehmend eigenständige Entscheidungen getroffen (Hurrelmann und Bauer 2020). Wünschenswert wäre an dieser Stelle eine Überprüfung der dargelegten Ergebnisse für Kinder jüngerer Altersgruppen. Zu welchem Zeitpunkt welche medienerzieherischen Strategien zum Einsatz kommen und welche Veränderungen sich bezüglich der Medienerziehung im jüngeren Kindes- und Jugendalter zeigen, scheinen für die medienpädagogische Forschung essenzielle 
Fragestellungen, denen weiter nachgegangen werden sollte. Eine konsistente oder inkonsistente Umsetzung von Regeln scheint sich dagegen als relativ stabiles Merkmal der Eltern-Kind-Kommunikation zu zeigen bzw. in der allgemeinen erzieherischen Praxis begründet zu sein. Die vorliegenden Ergebnisse zur Entwicklung der Subskalen der aktiven, restriktiven und technischen Mediation, sowie des Monitorings und des inkonsistenten Medienerziehungsverhaltens über vier Erhebungswellen hinweg können die bisherigen längsschnittlichen Erkenntnisse um einen entscheidenden neuen Einblick erweitern.

Die berichteten Ergebnisse und Implikationen müssen insbesondere vor dem methodischen Hintergrund der VEIF-Studie hinterfragt werden. So wird in der Studie ein besonderer Fokus auf exzessive Internetnutzung gelegt, der auch eine Verzerrung des Samples beinhaltet. Zwar sind nur bei einigen Subskalen und Erhebungswellen signifikante Unterschiede der medienerzieherischen Praktiken sowie der Erfassung inkonsistenter Medienerziehung zwischen Test- und Kontrollgruppe zu finden, allerdings sollten die bestehenden Unterschiede nicht vernachlässigt und in weiteren Analysen eingehender überprüft werden. Darüber hinaus muss auch die eher niedrige Reliabilität der Subskala zur aktiven Mediation bei der Betrachtung der Ergebnisse reflektiert werden. Eine Überarbeitung der Skala in deutscher Sprache und damit eine Weiterentwicklung hin zu einem reliableren Messinstrument wäre für eine künftige Betrachtung der Parental Mediation wünschenswert.

Der abnehmende Einsatz medienerzieherischer Praktiken im Jugendalter, verdeutlicht nichtsdestotrotz die Notwendigkeit entsprechende regulierende, sowie unterstützende Verhaltensweisen früh in Familien zu etablieren, um Kompetenzen im Umgang mit digitalen Medien bei Heranwachsenden fördern und negativen Aspekten der kindlichen Mediennutzung vorbeugen zu können. Denn wenn sich medienerzieherische Praktiken nicht bereits früh in der elterlichen Medienerziehung wiederfinden - sei es in der restriktiven, aktiven, technischen Mediation oder dem Monitoring -, so scheinen mögliche Förder- und Unterstützungsmöglichkeiten mit zunehmendem Alter kaum noch gegeben. 


\section{Literatur}

Autorengruppe Bildungsberichterstattung. 2020. Bildung in Deutschland 2020: Ein indikatorengestützter Bericht mit einer Analyse zu Bildung in einer digitalisierten Welt. Bielefeld: wbv. https://doi.org/10.3278/6001820gw.

Blum-Ross, Alicia, und Sonia Livingstone. 2016. Families and screen time: Current advice and emerging research. Media Policy Brief 17. London: Media Policy Project, London School of Economics and Political Science.

Cabello-Hutt, Tania, Patricio Cabello, und Magdalena Claro. 2018. "Online opportunities and risks for children and adolescents: The role of digital skills, age, gender and parental mediation in Brazil». New Media \& Society 20 (7): 2411-31. https://doi. org/10.1177/1461444817724168.

Çankaya, Serkan, und Hatice Ferhan Odabaşı. 2009. "Parental controls on children's computer and Internet use». Procedia - Social and Behavioral Sciences 1 (1): 1105-9. https://doi. org/10.1016/j.sbspro.2009.01.199.

Chng, Grace S., Dongdong Li, Albert K. Liau, und Angeline Khoo. 2015. «Moderating Effects of the Family Environment for Parental Mediation and Pathological Internet Use in Youths». Cyberpsychology, behavior and social networking 18 (1): 30-36. https://doi.org/10.1089/cyber.2014.0368.

Dedkova, Lenka, und David Smahel. 2020. «Online Parental Mediation: Associations of Family Members' Characteristics to Individual Engagement in Active Mediation and Monitoring». Journal of Family Issues 41 (8): 1112-36. https://doi.org/10.1177/0192513X19888255.

Dürager, Andrea, und Nathalie Sonck. 2014. Testing the reliability of scales on parental internet mediation. London: LSE. http://eprints.Ise.ac.uk/id/eprint/60220.

Eggert, Susanne, und Ulrike Wagner. 2016. Grundlagen zur Medienerziehung in der Familie. Studie. Expertise: MoFam - Mobile Medien in der Familie I. https://doi.org/10.25656/01:16560.

Feierabend, Sabine, Theresa Plankenhorn, und Thomas Rathgeb. 2017. «FIM-Studie 2016. Familie, Interaktion, Medien Untersuchung zur Kommunikation und Mediennutzung in Familien». Herausgegeben von Medienpädagogischer Forschungsverbund Südwest (mpfs). Medienpädagogischer Forschungsverbund Südwest (LFK, LMK). http://www.mpfs.de/fileadmin/files/Studien/KIM/2016/KIM_2016_Web-PDF.pdf.

Fu, Xinchen, Jingxuan Liu, Ru-De Liu, Yi Ding, Wei Hong, und Shuyang Jiang. 2020. «The impact of parental active mediation on adolescent mobile phone dependency: A moderated mediation model». Computers in Human Behavior 107:106280. https://doi.org/10.1016/j. chb.2020.106280.

Hasebrink, Uwe, und Sascha Hölig. 2017. «Deconstructing Audiences in Converging Media Environments». In Media Convergence and Deconvergence, herausgegeben von Sergio Sparviero, Corinna Peil, und Gabriele Balbi, 113-33. Cham: Springer. https://doi.org/10.1007/9783-319-51289-1_6. 
Hasebrink, Uwe, Claudia Lampert, und Kira Thiel. 2019. Online-Erfahrungen von 9- bis 17-Jährigen: Ergebnisse der EU Kids Online-Befragung in Deutschland 2019. 2. Auflage, revidierte Ausgabe. Hamburg: Hans-Bredow-Institut. https://www.hans-bredow-institut.de/uploads/media/Publikationen/cms/media/odfzzoy_EUKO_DE_191209.pdf.

Hepp, Andreas. 2018. «Von der Mediatisierung zur tiefgreifenden Mediatisierung». In Kommunikation - Medien - Konstruktion, herausgegeben von Jo Reichertz, und Richard Bettmann, 27-45. Wiesbaden: Springer Fachmedien Wiesbaden. https://doi.org/10.1007/978-3-65821204-9_2.

Hepp, Andreas. 2020. Deep mediatization. London: Routledge. https://doi.org/10.1080/15205 436.2020 .1856592$.

Hurrelmann, Klaus, und Ullrich Bauer. 2020. Einführung in die Sozialisationstheorie: Das Modell der produktiven Realitätsverarbeitung. 13. Auflage. Pädagogik. Weinheim: Beltz.

Iske, Stefan. 2012. «Medienerziehung». In Handbuch Erziehung, herausgegeben von Uwe Sandfuchs, 682-86. Bad Heilbrunn: Klinkhardt.

Kalmus, Veronika, Lukas Blinka, und Kjartan Ólafsson. 2015. «Does It Matter What Mama Says: Evaluating the Role of Parental Mediation in European Adolescents' Excessive Internet Use». Child Soc 29 (2): 122-33. https://doi.org/10.1111/chso.12020.

Kamin, Anna-Maria, und Dorothee M. Meister. 2020. «Familie und Medien». In Handbuch Familie, herausgegeben von Jutta Ecarius, und Anja Schierbaum, 1-19. Wiesbaden: Springer. https://doi.org/10.1007/978-3-658-19416-1_17-1.

Kammerl, Rudolf, Lena Hirschhäuser, Moritz Rosenkranz, Christiane Schwinge, Sandra Hein, Lutz Wartberg, und Kay Uwe Petersen. 2012. EXIF - Exzessive Internetnutzung in Familien: Zusammenhänge zwischen der exzessiven Computer- und Internetnutzung Jugendlicher und dem (medien-)erzieherischen Handeln in den Familien. Lengerich: Pabst Science Publishers.

Kammerl, Rudolf, und Michaela Kramer. 2016. «The Changing Media Environment and Its Impact on Socialization Processes in Families». Studies in Communication Sciences 16 (1): 21-27. https://doi.org/10.1016/j.scoms.2016.04.004.

Kammerl, Rudolf, Matthias Zieglmeier, und Lutz Wartberg. 2020. «Medienerziehung und familiale Aspekte als Prädiktoren für problematischen jugendlichen Internetgebrauch». Zeitschrift für Erziehungswissenschaft 23 (1): 175-91. https://doi.org/10.1007/s11618-01900920-1.

Kerr, Margaret, Håkan Stattin, und William J. Burk. 2010. «A Reinterpretation of Parental Monitoring in Longitudinal Perspective». Journal of Research on Adolescence 20 (1): 39-64. https://doi.org/10.1111/j.1532-7795.2009.00623.x.

Krotz, Friedrich. 2017. «Mediatisierung: Ein Forschungskonzept». In Mediatisierung als Metaprozess, herausgegeben von Friedrich Krotz, Cathrin Despotović, und Merle-Marie Kruse, 13-32. Wiesbaden: Springer Fachmedien Wiesbaden. https://doi.org/10.1007/978-3-65816084-5_2.

Lee, Sook-Jung. 2013. «Parental restrictive mediation of children's internet use: Effective for what and for whom?». New Media \& Society 15 (4): 466-81. https://doi. org/10.1177/1461444812452412. 
Livingstone, Sonia, und Ellen J. Helsper. 2008. «Parental Mediation of Children's Internet Use». Journal of Broadcasting \& Electronic Media 52 (4): 581-99. https://doi. org/10.1080/08838150802437396.

Mutlu-Bayraktar, Duygu, Özgür Yılmaz, und Gamze İnan-Kaya. 2018. «Digital Parenting: Perceptions on Digital Risks». kalem 14 (1): 137-63. https://doi.org/10.23863/kalem.2018.96.

Nathanson, Amy I. 1999. «Identifying and Explaining the Relationship Between Parental Mediation and Children's Aggression». Communication Research 26 (2): 124-43. https://doi. org/10.1177/009365099026002002.

Nikken, Peter, und Hanneke de Graaf. 2013. «Reciprocal Relationships Between Friends' and Parental Mediation of Adolescents 'Media Use and Their Sexual Attitudes and Behavior». Journal of youth and adolescence 42 (11): 1696-1707. https://doi.org/10.1007/s10964-0129873-5.

Padilla-Walker, Laura M., Sarah M. Coyne, Ashley M. Fraser, W. Justin Dyer, und Jeremy B. Yorgason. 2012. «Parents and Adolescents Growing up in the Digital Age: Latent Growth Curve Analysis of Proactive Media Monitoring». Journal of adolescence 35 (5): 1153-65. https:// doi.org/10.1016/j.adolescence.2012.03.005.

Paus-Hasebrink, Ingrid, Jasmin Kulterer, und Philip Sinner. 2019. Social Inequality, Childhood and the Media. Cham: Springer International Publishing. https://doi.org/10.1007/978-3030-02653-0.

Peled, Yehuda. 2018. «Children's Attitudes to Parental Mediation in a Traditional Society». Cyberpsychology, behavior and social networking 21 (12): 774-80. https://doi.org/10.1089/ cyber.2018.0165.

Pfetsch, Jan. 2018. «Jugendliche Nutzung digitaler Medien und elterliche Medienerziehung Ein Forschungsüberblick». Praxis der Kinderpsychologie und Kinderpsychiatrie - Ergebnisse aus Psychotherapie, Beratung und Psychiatrie 67 (2): 110-33. https://doi.org/10.13109/ prkk.2018.67.2.110.

Schaan, Violetta K., und André Melzer. 2015. «Parental Mediation of Children's Television and Video Game Use in Germany: Active and Embedded in Family Processes». Journal of Children and Media 9 (1): 58-76. https://doi.org/10.1080/17482798.2015.997108.

Schorb, Bernd, und Ulrike Wagner. 2013. «Medienkompetenz - Befähigung zur souveränen Lebensführung in einer mediatisierten Gesellschaft». In Medienkompetenzförderung für Kinder und Jugendliche: Eine Bestandsaufnahme, herausgegeben von Bernward Hoffmann, Dagmar Hoffmann, Kai-Uwe Hugger, Rudolf Kammerl, Dorothee M. Meister, Norbert Neuß, Ida Pöttinger et al., 18-23. Berlin: Bundesministerium für Familie, Senioren, Frauen und Jugend.

Shelton, Karen K., Paul J. Frick, und Jane Wootton. 1996. «Assessment of parenting practices in families of elementary school-age children». Journal of Clinical Child Psychology 25 (3): 317-29. https://doi.org/10.1207/s15374424jccp2503_8.

Sonck, Nathalie, Peter Nikken, und Jos de Haan. 2013. «Determinants of Internet Mediation». Journal of Children and Media 7 (1): 96-113. https://doi.org/10.1080/17482798.2012.7398 06. 
Spanhel, Dieter. 2006. Medienerziehung: Erziehungs- und Bildungsaufgaben in der Mediengesellschaft. Handbuch Medienpädagogik. Stuttgart: Klett-Cotta.

Spanhel, Dieter. 2015. «Der Prozess der Medienbildung bei Kindern und Jugendlichen und seine Ausrichtung durch Medienerziehung». medienimpulse 53 (2 Begründungen und Ziele der Medienbildung). https://doi.org/10.21243/mi-02-15-02.

Symons, Katrien, Koen Ponnet, Michel Walrave, und Wannes Heirman. 2017. «A qualitative study into parental mediation of adolescents' internet use». Computers in Human Behavior 73:423-32. https://doi.org/10.1016/j.chb.2017.04.004.

Tulodziecki, Gerhard. 2017. «Medienerziehung». In Grundbegriffe Medienpädagogik, herausgegeben von Bernd Schorb, Anja Hartung-Griemberg, und Christine Dallmann. 6., neu verf. Aufl., 234-40. München: kopaed.

Tulodziecki, Gerhard, Bardo Herzig, und Silke Grafe. 2019. Medienbildung in Schule und Unterricht: Grundlagen und Beispiele. 2. Aufl. Bad Heilbrunn: UTB.

Valkenburg, Patti M., Marina Krcmar, Allerd L. Peeters, und Nies M. Marseille. 1999. «Devloping a Scale to Assess Three Styles of Television Mediation: Instructive Mediation, Restrictive Mediation, and Social Coviewing». Journal of Broadcasting \& Electronic Media 43.

Wagner, Ulrike, Susanne Eggert, und Gisela Schubert. 2016. MoFam - Mobile Medien in der Familie. Studie. Langfassung. https://www.jff.de/fileadmin/user_upload/jff/projekte/mofam/ JFF_MoFam1_gesamtStudie.pdf.

Wagner, Ulrike, Christa Gebel, und Claudia Lampert, Hrsg. 2013. Zwischen Anspruch und Alltagsbewältigung: Medienerziehung in der Familie 72. Berlin: Vistas.

Wahyuningrum, Enjang, Suryanto, und Dewi Retno Suminar. 2020. «Parenting in Digital Era: a Systematic Literature Review». Journal of Educational, Health and Community Psychology 9 (3): 226-58. https://doi.org/10.12928/jehcp.v9i3.16984.

Wößmann, Ludger, Vera Freundl, Elisabeth Grewenig, Philipp Lergetporer, Katharina Werner, und Larissa Zierow. 2021. "Bildung erneut im Lockdown: Wie verbrachten Schulkinder die Schulschließungen Anfang 2021?». ifo Schnelldienst 74 (05): 36-52. https://www.ifo.de/ publikationen/2021/aufsatz-zeitschrift/bildung-erneut-im-lockdown-wie-verbrachtenschulkinder-die. 